\title{
Numerical equation for the mass stopping power of protons in human body subtances
}

\author{
Eko Sulistya $^{1, a}$, Kusminarto $^{1}$, Arief Hermanto ${ }^{1}$ \\ Department of Physics, Faculty of Mathematics and Natural Sciences \\ Universitas Gadjah Mada \\ Yogyakarta, Indonesia \\ asulistya@ugm.ac.id
}

\begin{abstract}
Accurate determination of the protons energy in radiotherapy planning can be achieved if the stopping power of protons to specific body tissue are known. The Bethe-Bloch equation greatly aid the understanding of how to calculate the stopping power from the physics point of view, but not so practical and easy to be applied on radiotherapy planning system that involves many computational calculations and computer programming. In this paper we present a numerical equation for mass stopping power of protons in four types of human body substance, i.e. water, bone, muscle and tissue. The equation was obtained by fitting the data values obtained from SRIM program. By using the numerical equation, then the algorithm programming is expected to become simpler and easier.
\end{abstract}

Keywords - radiotheraphy; proton; stopping power; human tissue; numerical;

\section{INTRODUCTION}

Radiotherapy is one of the important methods in the treatment of cancer. Radiotherapy with proton beam has several advantages compared with radiotherapy with X-rays (high energy photons). After a short build-up region, Xrays radiation shows an exponentially decreasing energy deposition with increasing depth in the medium. In contrast, protons show an increasing energy deposition with penetration distance and leading to a maximum, called the "Bragg peak", near the end of range of the proton beam [1]. The energy of proton then can be set so that the Bragg peak occurs at the cancer location. Thus the cancer tissue receive doses much higher than the healthy tissue around it. Radiotherapy with proton beam has been done and the results indicate that treatment with proton beam does have clinical advantages compared with conventional therapy with $\mathrm{X}$-rays [2][3][4][5].

Determination of proton energy corresponding to the location of the cancer become an important requirement in proton radiotherapy. The Bragg peak is very narrow so if shifted from the desired target will couse healthy tissue to exposed very high doses. The location where proton will stopped is closely related to the amount of stopping power of the medium through which it passes, which is a measure of how much energy is lost given to the medium. The magnitude of the stopping power depends on the proton energy and the type of the target medium. By definition, stopping power is energy loss per unit of distance traveled in the target medium. So by knowing the stopping power of protons in the medium, the position of the proton can be predicted.

In the radiotherapy with human body, medium that should be considered is water, bones, muscles, and tissues.

\section{THEORY, RESULT AND DISCUSSION}

Equation of the stopping power for an heavy ion in a homogeneous medium was obtained by Bethe using quantum mechanics [6],

$-\frac{d E}{d x}=\frac{4 \pi k_{0}^{2} z^{2} e^{4} n}{m c^{2} \beta^{2}}\left[\ln \frac{2 m c^{2} \beta^{2}}{I\left(1-\beta^{2}\right)}-\beta^{2}\right]$

where $k_{0}=8.99 \times 10^{9}$ N.m $. \mathrm{C}^{2}, z=$ atomic number of heavy ion, $e=$ electron charge, $n=$ number of electrons per unit volume of the medium, $m=$ electron rest mass, $c$ $=$ speed of light in vacuum, $\beta=$ the ratio of the speed of ion to the speed of light, and $I=$ mean excitation energy of the medium. Equation (1) is not valid for low-energy ions, when the ion velocity is much smaller than the speed of the electron orbit. For energies smaller than $25 \mathrm{keV}$, stopping power equation was proposed by Fermi and Teller, and for energies above $100 \mathrm{keV}$, Bohr proposed an equation based on the Thomas-Fermi atomic model [7].

In designing a computer software that will be used in proton radiotherapy planning, it would be easier if there is an equation that is applicable to all energy regions. If that equation does not exist then the code will be longer and more complicated because each step the program must decide which equation to be used according to the proton energy. Singh [8] propose an empirical equation to obtain the mass stopping power for protons at $0.5 \mathrm{Mev}$ to $200 \mathrm{MeV}$ energy range in biological human body substances such as water, bone, muscle and tissue. Empirical equation proposed by Singh is

mass stopping power $=y_{0}+A_{1} e^{\left(-\frac{x}{t_{1}}\right)}$

where $y_{0}, A_{1}$ and $t_{1}$ are constants obtained from the exponential fitting of empirical data. The value of $x=$ $\langle Z / A\rangle E^{0.05}$ depends on atomic number $(Z)$, atomic weight $(A)$ and energy $(E)$. 
The result of the calculation (2) agreed with the results of calculations performed by Mukherjee [9]. Both Singh and Mukherjee et al did not give the value of stopping power of protons to energies below $0.5 \mathrm{MeV}$.

With the SRIM software (Stopping and Range of Ions in Matter) we can obtain the stopping power value for any ion in any material and proton energy in the range $10 \mathrm{eV}-2$ $\mathrm{GeV}$. The programs were developed by James F. Ziegler and Jochen P. Biersack around 1983 [10][11] and are being continuously upgraded with the major changes occurring approximately every five years. SRIM is based on a Monte Carlo simulation method, namely the binary collision approximation with a random selection of the impact parameter of the next colliding ion [12]. As the input parameters, it needs the ion type and energy and the material of one or several target layers.

Fig. 1 shows the output of the program SRIM for 4 materials, i.e. water, bone, muscle and tissue. The curves depict the relationship between proton energy ( $\mathrm{H}$ ions) with the value of mass stopping power in units of $\mathrm{MeV} /\left(\mathrm{g} / \mathrm{cm}^{2}\right)$.

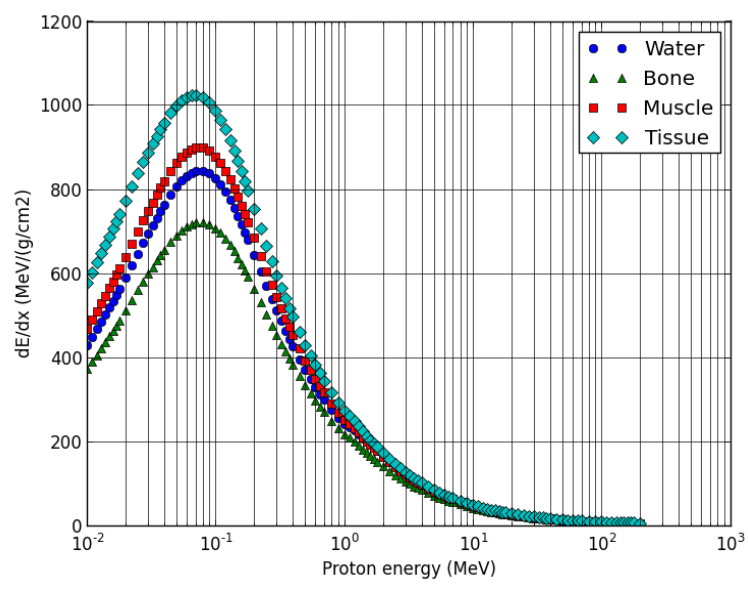

Figure 1. Mass stopping power of proton in 4 human body substances obtained with SRIM program

Any type of equation can be fitted to the data points in Fig.1. We found that the equation which is best suited to represent the data points is non-linear equation, as shown in (3). $E$ is the proton energy, while $a, b, c, d, e, f, g$ and $h$ are the fitting parameters whose values depend on the type of material.

$$
\frac{d E}{d x}=\frac{a+c \ln E+e(\ln E)^{2}+g(\ln E)^{3}}{1+b \ln E+d(\ln E)^{2}+f(\ln E)^{3}+h(\ln E)^{4}}
$$

Fitting parameters for water, bone, muscle and tissue are presented in Table I. In the SRIM materials target library, there are several types of bones, muscles, and tissues of the body, which is determined by the percentage composition of the constituent atoms. We select one of the types of targets that represent bone, muscles, and tissues, and is written at the bottom of the Table I.
TABLE I. FIT PARAMETERS FOR WATER, BONES, MusCle, AND TISSUE

\begin{tabular}{|c|r|r|r|r|}
\hline \multirow{2}{*}{ Parameters } & \multicolumn{5}{|c|}{ Subtances } \\
\cline { 2 - 5 } & \multicolumn{1}{|c|}{ Water } & \multicolumn{1}{c|}{ Bone $^{*}$} & Muscle $^{* *}$ & Tissue $^{* * * *}$ \\
\hline$a$ & 244.830 & 217.957 & 253.616 & 272.416 \\
\hline$b$ & -0.727 & -0.299 & -0.726 & -0.688 \\
\hline$c$ & -306.162 & -196.901 & -335.142 & -365.781 \\
\hline$d$ & 0.504 & 0.102 & 0.366 & -0.001 \\
\hline$e$ & 275.476 & 102.148 & 258.892 & 184.766 \\
\hline$f$ & 0.416 & 0.125 & 0.335 & 0.127 \\
\hline$g$ & -41.632 & -13.032 & -38.520 & -24.067 \\
\hline$h$ & 0.116 & 0.044 & 0.098 & 0.050 \\
\hline
\end{tabular}

Equation (3) can be easily implemented in any programming language, without having to take into account the atomic number and atomic mass of the target medium. By using the values of the parameters in Table I, we calculated value of the mass stopping power for 4 substances the human body for proton energies between $0.5 \mathrm{MeV}$ to 200 $\mathrm{MeV}$ and compared with the results of Mukherjee and Singh. The calculated value are presented in Figure 2, Figure 3, Figure 4 and Figure 5, respectively for water, bone, muscle, and tissue.

All four graphs show that the results of calculations with (3) suitable for proton energy above $2 \mathrm{MeV}$. At low energy the nuclear stopping is dominant, while (3) based on the data of electronic stopping. SRIM implemented Core and Bond corrections on the interaction of ions with atoms and electrons of the target, which can also affect the difference between the results of calculations for low energy. However, differences in the results for low energy may also be caused by other factors which should be investigated further.

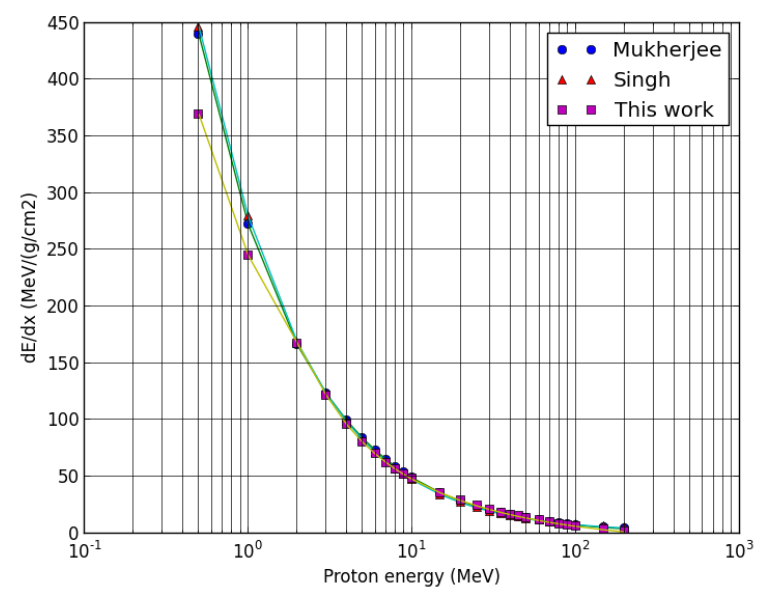

Figure 2. Comparison of three calculations of mass stopping power of proton in water 


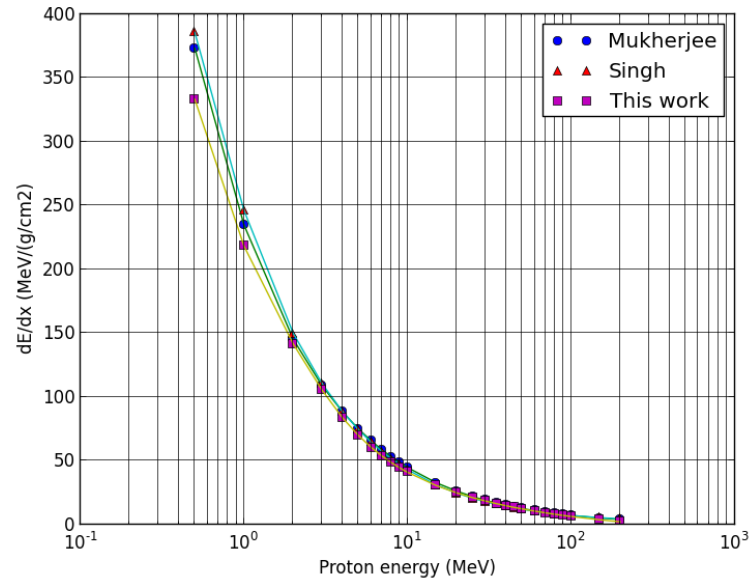

Figure 3. Comparison of three calculations of mass stopping power of proton in bone

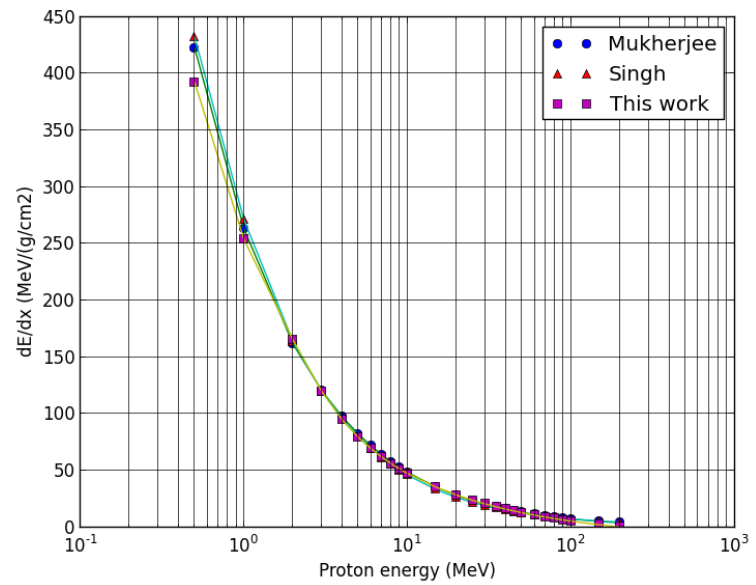

Figure 4. Comparison of three calculations of mass stopping power of proton in muscle

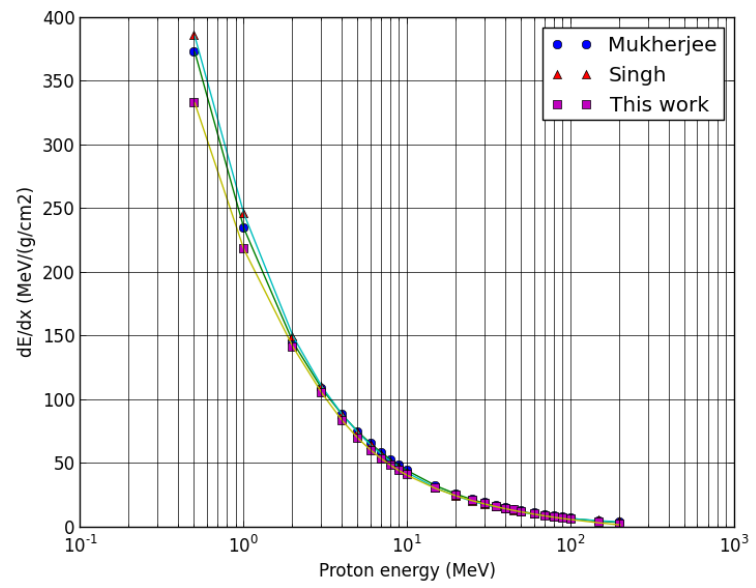

Figure 5. Comparison of three calculations of mass stopping power of proton in tissue
Proton energy used in the radiotherapy are generally on the order of tens of $\mathrm{MeV}$ [11] [12]. In that order of proton energy, the calculation results on the mass stopping power of tissue for the three methods can be said to agree with each other.

\section{CONCLUSION AND SUGGESTIONS}

From the above results obtained using the proposed numerical equation (3), the mass stopping power of materials can be expressed in non-linear equation. The calculated mass stopping power is in aggreement with previously calculated value in high energy range. It may be noted also that the proposed numerical equation has a simple form and easy to implement in programming. Further investigation needs to be carried out at low proton energy, in order to obtain equations valid for all energy regions.

\section{REFERENCES}

[1] Paganetti, H., and Bortfeld, T., " Proton beam radiotherapy - the state of the art", Medical Radiology Series, New Technologies in Radiation Oncology, Heidelberg: Springer Verlag, 2005.

[2] De Cicco, L., Cella, L., Liuzzi, R., Solla, R., Farella, A., Punzo, G., and Pacelli, R., "Radiation therapy in primary orbital lymphoma: a single institution retrospective analysis", Radiat Oncol, 4, 60, December 2009, doi: 10.1186/1748-717X-4-60

[3] Devicienti, S., Strigari, L., D'Andrea, M., Benassi, M., Dimiccoli, V., and Portaluri, M.,"Patient positioning in the proton radiotherapy era", J Exp Clin Cancer Res, 29, 47, 2010, doi: 10.1186/1756-9966-29-47

[4] Habrand, J. L., Bolle, S., Datchary, J., Alapetite, C., Petras, S., Helfre, S., and Doz, F.,"Proton beam therapy in pediatric radiotherapy". Cancer/Radiothérapie, 13(6-7), 2009, pp. 550-555.

[5] Hillbrand, M., Georg, D., Gadner, H., Potter, R., and Dieckmann, K., "Abdominal cancer during early childhood: a dosimetric comparison of proton beams to standard and advanced photon radiotherapy", Radiotheraphy Oncol, 89(2), 2008, pp. 141-149

[6] Turner, J. E., "Atoms, radiation, and radiation protection", $3^{\text {rd }}$ ed, WILEY-VCH Verlag GmbH and Co. KGaA, 2007.

[7] Getachew, A., "Stopping power and range of protons of various energies in different materials", Department of Physics, Ethiopia, Addis Ababa University, Master of Science in Physics: 40, 2007

[8] Singh, H., S. K. Rathi, and A. S. Verma. "Stopping powers of protons in biological human body substances", Universal Journal of Medical Science, 2013, pp. 17-22, doi: 10.13189/ujmsj.2013.010201

[9] Mukherjee B., "Mass stopping powers of protons up to $200 \mathrm{MeV}$ in some biologically important materials", Nucl. Inst. Meth. 1983; 211: pp. 235-237.

[10] J. F. Ziegler, J. P. Biersack and U. Littmark, "The stopping and range of ions in solids", New York: Pergamon Press, 1996

[11] Ziegler, J.F, J.P Biersack, and M.D. Ziegler, "SRIM - The Stopping and Range of Ions in Matter", SRIM Co., Chester, Maryland, USA, 2008

[12] Mowlavi, A.A., Homaei, M., and Alipoor, M., "SRIM Code Use for the Evaluation of Optimum Proton Energy Interval for Thyroid Cancer Therapy", African Physical Review, 107, 2010

[13] Sulistya, E., Kusminarto, and Hermanto A., "Computation to obtain the spread out bragg peak (SOBP) for proton radiotherapy on model of thyroid cancer", Proceeding of International Conference On Research, Implementation And Education Of Mathematics And Sciences, Yogyakarta State University, P13-P17, 2014 